\title{
Entre la negación y la impotencia: prestadores de servicios de salud ante la violencia contra las mujeres en México
}

\author{
Cristina Herrera, M en Cs Soc, (1) Ari Rajsbaum, MC, ${ }^{(2)}$ \\ Carolina Agoff, Dr en Cs Soc, ${ }^{(3)}$ Aurora Franco, Lic en Tr Soc. ${ }^{(4)}$
}

\begin{abstract}
Herrera C, Rajsbaum A, Agoff C, Franco A.
Entre la negación y la impotencia: prestadores de servicios de salud ante la violencia contra las mujeres en México. Salud Publica Mex 2006;48 supl 2:S259-S267.
\end{abstract}

\begin{abstract}
Resumen
Objetivo. Conocer los significados y prácticas respecto a la violencia doméstica entre prestadores de servicios de salud, para mejorar la atención. Material y métodos. Entre mayo y noviembre de 2003 se aplicaron 60 entrevistas en profundidad a prestadores de servicios públicos de salud y a informantes clave, en unidades de atención en tres entidades de alta prevalencia de violencia doméstica Quintana Roo, Coahuila y el Distrito Federal. Resultados. Los prestadores fueron clasificados por su grado de adhesión a cuatro tipos de "discursos sobre la violencia" que orientan su práctica cotidiana y que están relacionados con sus valores de género y sus posiciones sociales. La mayoría manifestó disposición a atender el problema. Conclusiones. La atención de la violencia doméstica es insuficiente en los servicios de salud, pero esto parece deberse más a que el discurso tolerante de la violencia predominó entre los tomadores de decisiones, que a las actitudes de los propios prestadores de servicios; éstos demostraron mayor conciencia de los derechos de las mujeres.
\end{abstract}

Palabras clave: violencia doméstica; género; prestadores de servicios de salud; México
Herrera C, Rajsbaum A, Agoff C, Franco A.

Between denial and helplessness: health care providers

facing domestic violence in Mexico.

Salud Publica Mex 2006;48 suppl 2:S259-S267.

\begin{abstract}
Objective. To increase the knowledge of health care providers' understanding of and practices on domestic violence, in order to improve care. Materials and Methods. This study was conducted between May and November 2003, In Quintana Roo, Coahuila, and Mexico City, three Mexican states with a high prevalence of domestic violence. Sixty indepth interviews with health care providers in public health institutions and key informants of health service provision were completed. Results. Health care providers were categorized based on the extent to which they reproduced four "discourses about violence". These discourses influence their daily practice and are related to the informants' gender values and social positions. Most informants expressed their willingness to address the issue of domestic violence. Conclusions. Attention to and care for domestic violence is insufficient in health services; this may be due more closely related to a predominant discourse tolerating domestic violence among decision-makers, than to the attitudes of health care providers; the latter demonstrated greater awareness of women's rights.
\end{abstract}

Keywords: domestic violence; gender; health care providers; Mexico

(I) Centro de Investigaciones en Sistemas de Salud, Instituto Nacional de Salud Pública. México.

(2) Clínica de Atención a la Violencia Doméstica. Instituto Latinoamericano de Estudios de la Familia. México.

(3) Centro Regional de Investigaciones Multidisciplinarias (UNAM). México.

(4) Centro de Encuestas. Instituto Nacional de Salud Pública. México.

Fecha de recibido: 26 de agosto de 2004 - Fecha de aprobado: 24 de mayo de 2005 Solicitud de sobretiros: Dra. Carolina Agoff. Centro Regional de Investigaciones Multidisciplinarias (UNAM). Av. Universidad s/n, circuito 2, 62210 Cuernavaca, Morelos. Correo electrónico: carolina.agoff@gmx.de 
E n años recientes, la violencia intrafamiliar ha ganado un mayor reconocimiento en el discurso político como problema de salud pública. Sin embargo, por largo tiempo se ha ocultado debido a que se vive como un problema privado, con frecuencia vergonzante, pero al mismo tiempo como parte de la "normalidad" de las relaciones conyugales. ${ }^{1}$ El supuesto de partida fue que esta visión, según la cual la violencia de pareja es algo difícil de aceptar pero "normal", es compartida por la mayor parte de los servidores públicos encargados de dar atención al problema. Esto resulta ser un círculo vicioso de ocultamiento-normalización y reproducción de la cultura que da sustento a la violencia que es, probablemente, una de las principales razones por las cuales la violencia de pareja no ha sido percibida ni atendida de manera adecuada en los servicios encargados de dar atención. Dada su particular ubicación, los prestadores de salud son actores clave en el proceso de prevención, detección, atención y seguimiento de los problemas originados en la violencia intrafamiliar. Empero, este sector no ha respondido de manera efectiva, como se informa también en otras partes del mundo. ${ }^{*, 2,3}$

En un estudio entre médicos de primer nivel de atención en los Estados Unidos de América, por ejemplo, se encontraron, como barreras para una adecuada atención a la violencia, la identificación cercana con las pacientes, el miedo a ofender, la falta de entrenamiento y la falta de tiempo. ${ }^{4}$ Otros estudios han mostrado que, dado que el personal de salud es producto de la misma tradición cultural, no necesariamente juzga de manera negativa las actitudes que dan soporte al abuso contra la mujer. ${ }^{5}$ La actitud de los profesionales de la salud hacia la violencia puede ser potencialmente perjudicial, y esto se ha observado en mujeres que señalan haber sido doblemente victimizadas: por parte del abusador, y también por parte del personal de salud que las responsabiliza del maltrato sufrido. ${ }^{\ddagger}, 6-8$

Los factores que contribuyen a generar esta dificultad de percepción y atención de la violencia son de diversa índole y abarcan niveles tanto micro como macrosociales. Entre los primeros, se ubican los factores de tipo personal que se ponen en juego en la interacción entre los agentes involucrados, que en este caso son el personal de los servicios y las usuarias de los

\footnotetext{
* Rodríguez-Bolaños R, Márquez-Serrano M, Kageyama- Escobar ML. Violencia de género: actitud y conocimiento del personal de salud de Nicaragua. En prensa.

‡ La referencia 6 puede consultarse en las páginas S307-S314 en este mismo suplemento.
}

mismos. ${ }^{*}$, Entre los factores macrosociales, los más prominentes son los aspectos culturales y sociales derivados del sistema de sexo-género ${ }^{10}$ dominante y de las representaciones sociales a él vinculadas -se ha llamado la atención sobre el hecho de que los profesionales de salud son, ante todo, personas, y que, como tales, comparten los mismo valores culturales y a veces viven los mismos niveles de violencia que aquellas pacientes a quienes deben tratar y dar apoyo- ${ }^{11} \mathrm{y}$, finalmente, los factores de tipo político e institucional relacionados con la manera en que el problema es definido en el espacio público y tratado en el proceso del diseño de programas y políticas.

Los resultados que aquí se notifican forman parte de los principales hallazgos de un estudio cualitativo diseñado para explorar en profundidad y ampliar algunos de los aspectos cubiertos por la Encuesta Nacional sobre Violencia contra las Mujeres 2003 (ENVIM 2003). ${ }^{12}$ El objetivo de este estudio fue analizar en profundidad la manera en que perciben y enfrentan la violencia los principales actores involucrados en este problema en los servicios de salud -las mujeres que sufren violencia y los prestadores que las reciben-, con el fin de comprender cómo sus valores y concepciones de género, la percepción de las causas y consecuencias de la violencia y los efectos de estas concepciones, conocimientos, percepciones y vivencias afectan la interacción entre ellos en los servicios de salud y dificultan la atención. En el caso de los prestadores, que son el objeto de este artículo, el análisis de los datos permitió revelar diferencias importantes en sus discursos y en las motivaciones que orientan sus prácticas, algunas de las cuales quedan ilustradas en el presente texto.

\section{Material y métodos}

Se diseñó un estudio cualitativo donde se aplicaron las siguientes técnicas de recolección de datos:

- 60 entrevistas en profundidad con prestadores de servicios de salud de las tres instituciones del sector [Instituto Mexicano del Seguro Social (IMSS), Instituto de Seguridad y Servicios Sociales de los Trabajadores del Estado (ISSSTE) y Secretaría de Salud (SSA)], en tres entidades federativas con alta prevalencia de violencia de pareja (Quintana Roo, Coahuila y el Distrito Federal). ${ }^{11}$ Se llevó a cabo un número equivalente de entrevistas a cada tipo

\footnotetext{
* Se ha documentado, por ejemplo, la dinámica potencial de abuso en la interacción enfermera-paciente.
} 
de profesional en los tres estados y se obtuvo la siguiente distribución: 16 médicos/as, 14 enfermeros/as, 11 psicólogos/as o psiquiatras y 19 trabajadores/as sociales;

- observación participante en los servicios de salud y diario de campo en las tres entidades; $y$

- $\quad$ entrevistas con informantes clave (autoridades del sector, directores/as de las unidades de atención, jefes de programas especiales y de servicios).

El propósito era indagar, a través de una guía de preguntas abiertas, los siguientes aspectos: sensibilidad ante el tema de la violencia de pareja y calidad de la atención que brindan a la mujer maltratada; canalización de casos a otras instancias y disposición a atestiguar en un proceso legal; conocimiento y aplicación de la norma oficial; concepciones sobre la violencia de pareja, sus causas y consecuencias; posición frente a la posibilidad de abortar legalmente en caso de violación; capacitación recibida sobre violencia de pareja.

Las entrevistas tuvieron una duración promedio de 50 minutos y las condujo y grabó en audio un equipo de cuatro investigadores formados en las áreas de sociología, psicología y psicología social.

El análisis de los datos se llevó a cabo según los principios de la teoría fundamentada. ${ }^{13,14} \mathrm{El}$ análisis generativo consiste, básicamente, en la elaboración de hipótesis emergentes a partir de los datos y en su constatación mediante el estudio comparativo de casos seleccionados en virtud de su valor informativo.

El Comité de Evaluación Ética del Instituto Nacional de Salud Pública aprobó el protocolo. De acuerdo con sus recomendaciones, sólo se entrevistó a los profesionales de salud que accedieron voluntariamente a participar en el estudio y en todos los casos se obtuvo el consentimiento informado de los individuos entrevistados, garantizándoles el anonimato. Las entrevistas se realizaron en el lugar de trabajo, en el espacio privado de un cubículo o consultorio.

\section{Resultados}

$\mathrm{Al}$ analizar las razones que los prestadores de salud esgrimen para justificar las deficiencias que existen en la atención de problemas de violencia, considerando tanto las percepciones que tienen de la misma como sus actitudes y sus sentimientos hacia las usuarias que la padecen, se encontró que, aun cuando los resultados de sus prácticas sean más o menos semejantes, existen notables diferencias entre sus argumentos y actitudes que tienen interesantes repercusiones para un eventual diseño de programas y políticas dirigidos a mejorar la atención del problema.
De manera congruente con lo señalado en otros estudios en el país, ${ }^{15-18}$ lo primero que llama la atención es la tendencia a no atender los casos de violencia de manera adecuada en los servicios de salud. Las prácticas cotidianas de los prestadores no se guían por lo que la norma oficial ${ }^{19}$ prescribe como rutina obligatoria en estos casos. El conocimiento de esta norma es casi nulo, por lo que ningún prestador utiliza sus hojas de registro y, en cambio, se suelen utilizar formatos de registro de accidentes o de lesiones. Una minoría de prestadores indicó haber recibido capacitación en el tema, la cual ha resultado útil para volver visible el problema y generar empatía con las víctimas, pero aun en esos casos, ha resultado insuficiente ya que los trabajadores manifiestan no saber cómo afrontar realmente el problema. La violencia suele pasarse por alto, y cuando se detecta, la mujer que la notifica es canalizada al servicio de trabajo social, donde no siempre se sabe hacia qué instancia enviarla, o bien, se tiene desconfianza en las instituciones indicadas para este fin. En la mayoría de los casos, salvo excepciones significativas como se verá, no existe un seguimiento posterior.

Con el objeto de comprender más a fondo los motivos de estas prácticas comunes, se elaboró un modelo de análisis basado en los datos recogidos, $\mathrm{y}$ a partir de ello, se construyeron cuatro tipos de "discurso" que permiten clasificar a los informantes y que ponen al descubierto las motivaciones que orientan sus prácticas profesionales. Las respuestas de los prestadores de salud a preguntas como ¿qué siente frente a una mujer maltratada?; ¿qué hace cuando se presenta un caso de maltrato en su servicio?; y ipor qué lo hace? podrían dividirse en dos niveles. El primero, de tipo axiológico, en el que se encuentran condensados una serie de discursos referentes particularmente a cuestiones de género y familia, y el segundo, pragmático, donde se ubican argumentos referentes a prácticas de tipo institucional y/o profesional.

\section{Discursos y prácticas relacionados con la violencia contra las mujeres}

En el nivel llamado axiológico, se encontró que las respuestas a las preguntas antes enunciadas diferían notablemente entre los prestadores entrevistados. Por ello, se clasificaron de acuerdo con el mayor o menor grado en que se adherían a uno de los cuatro tipos de "discursos", a los que se llamó:

1. Discurso dominante basado en estereotipos rígidos de género.

2. Discurso antiviolencia basado en valores tradicionales de género. 
3. Discurso antiviolencia basado en algunas ideas sobre "lo moderno" .

4. Discurso antiviolencia basado en los derechos de la mujer.

Estos tipos de discurso son construcciones teóricas y se elaboraron a partir de los datos empíricos, teniendo en cuenta las siguientes dimensiones de análisis:

1. Los valores de género predominantes en cada informante (clasificados según el eje tradicional -moderno).

2. Las concepciones de los distintos prestadores de salud sobre la violencia.

3. Los sentimientos y actitudes de estos profesionales frente a las usuarias que sufren violencia.

4. Las prácticas profesionales más habituales ante este problema en los servicios de salud.

Los cuatro tipos resultantes se resumen en el cuadro I.

\section{Discurso dominante basado en estereotipos rígidos} de género

A este grupo se le podría llamar los "negadores de la violencia". Esta categoría agrupa a quienes manifiestan tener valores tradicionales en relación con el géne- ro y la familia y que se adhieren a ellos sin cuestionar las diferencias jerárquicas y los roles tradicionalmente asignados a cada género (la mujer sumisa y dedicada principalmente al hogar vs. el hombre dominante y obligado a mantener a la familia), así como tampoco la desigualdad de poder subyacente. Este tipo de informantes tiende a negar o a restar importancia al problema con argumentos como "sufren más los niños y ancianos" o "las mujeres también son/somos violentas" etcétera. Suelen pasar por alto los problemas de las usuarias relacionados con la violencia o, en caso de ser visibles y explícitos, desconfiar de la versión que de ellos dan las mujeres. En este grupo, la negación, o bien, la estrategia de diluir la gravedad de la violencia contra las mujeres, forma parte de una retórica de no compromiso que los exime de cualquier responsabilidad frente al problema. Detrás suele haber una "naturalización" del problema, tal como expresó un alto funcionario de salud: "¡Por favor! ¿Quién no le ha gritado o ha insultado a su mujer en el último año?" Estos prestadores tienden a enjuiciar y culpabilizar a las mujeres víctimas de violencia, ya sea por ser agredidas ("ellas se lo buscan"), o bien, por ser agresoras (por ejemplo, de sus hijos). No consideran que la violencia doméstica sea un delito y, en cualquier caso, se desentienden de su obligación de dar seguimiento a las usuarias que la sufren, argumentando que es tarea de un médico legista. En suma, quienes comparten un

\section{Cuadro I}

Discursos y PRÁcticas de los PRESTAdores de SERVICIOS De SALUd ANTE LA VIOLENCIA CONTRA LAS MUJERES

\begin{tabular}{|c|c|c|c|c|}
\hline Valores predominantes & Tipo de discurso & Percepción de la violencia & Actitudes hacia la víctima & Prácticas típicas \\
\hline \multirow[t]{2}{*}{ Tradicionales } & $\begin{array}{l}\text { I. Dominante basado en } \\
\text { estereotipos rígidos } \\
\text { de género. }\end{array}$ & $\begin{array}{l}\text { La niega, diluye o minimiza; en } \\
\text { casos extremos, se burla. }\end{array}$ & $\begin{array}{l}\text { Indiferencia, ignorancia o } \\
\text { aceptación pasiva de la violen- } \\
\text { cia como algo "natural"; en } \\
\text { casos extremos, desprecio o } \\
\text { enjuiciamiento de la mujer. }\end{array}$ & $\begin{array}{l}\text { El médico/a trata la lesión, no indaga } \\
\text { en causas; si la mujer menciona la vio- } \\
\text { lencia, a veces canaliza a trabajo so- } \\
\text { cial; no seguimiento de los casos. }\end{array}$ \\
\hline & $\begin{array}{l}\text { 2. Antiviolencia basado } \\
\text { en valores tradiciona- } \\
\text { les de género. }\end{array}$ & $\begin{array}{l}\text { Existe como desviación del } \\
\text { ideal de armonía familiar ba- } \\
\text { sado en las obligaciones y vir- } \\
\text { tudes de cada uno de los gé- } \\
\text { neros. }\end{array}$ & $\begin{array}{l}\text { Pena ajena. La violencia es vis- } \\
\text { ta como un asunto doloroso } \\
\text { pero privado. } \\
\text { Identificación de género si la } \\
\text { prestadora es mujer. Impo- } \\
\text { tencia. }\end{array}$ & $\begin{array}{l}\text { Dependiendo de motivaciones indivi- } \\
\text { duales (tener "vocación" de psicólo- } \\
\text { go/a) indagan las causas y aconsejan a } \\
\text { la mujer o la canalizan; no seguimien- } \\
\text { to de los casos. }\end{array}$ \\
\hline \multirow[t]{2}{*}{ Modernos } & $\begin{array}{l}\text { 3. Antiviolencia basado } \\
\text { en algunas ideas sobre } \\
\text { "lo moderno". }\end{array}$ & $\begin{array}{l}\text { Existe por falta de educación } \\
\text { o "cultura", por origen rural o } \\
\text { indígena u otros por el estilo. }\end{array}$ & $\begin{array}{l}\text { Coraje hacia la víctima por } \\
\text { "dejarse"; en algunos casos } \\
\text { desprecio encubierto. Distan- } \\
\text { ciamiento social. }\end{array}$ & $\begin{array}{l}\text { Tienden a la pasividad pero a veces de } \\
\text { manera selectiva y dependiendo de } \\
\text { motivaciones personales, pueden } \\
\text { aconsejar terapias o canalizar a tra- } \\
\text { bajadora social y de ahí al ministerio } \\
\text { público. }\end{array}$ \\
\hline & $\begin{array}{l}\text { 4. Antiviolencia basado } \\
\text { en los derechos de la } \\
\text { mujer. }\end{array}$ & $\begin{array}{l}\text { Existe por desigualdad de po- } \\
\text { der e inequidad entre géneros. }\end{array}$ & $\begin{array}{l}\text { Indignación ante una injusticia; } \\
\text { identificación con la víctima si } \\
\text { la prestadora es mujer. }\end{array}$ & $\begin{array}{l}\text { Disposición a atender el caso, diver- } \\
\text { sos grados de involucramiento; inten- } \\
\text { to de empoderar; impotencia por fal- } \\
\text { ta de apoyo; a veces acompañan a la } \\
\text { mujer a denunciar. }\end{array}$ \\
\hline
\end{tabular}


universo de valores donde la violencia hacia las mujeres es tolerada como algo "normal" tienden a ignorar el problema cuando aparece en los servicios, a no darle canalización y, en el peor de los casos, a dar a las usuarias que lo padecen un trato humillante, con lo que las maltratan en segunda instancia. Los informantes que se adscriben a este discurso son, en su mayoría, hombres, y representan a todas las profesiones incluidas en la muestra. En este grupo se ubicó también a algunos profesionales con posiciones jerárquicas tales como autoridades del sector, directores de hospitales, encargados de programas especiales, jefes de servicios, etcétera. Estos profesionales no fueron informantes directos, sino que actuaron como informantes clave o intermediarios, facilitando el contacto con los informantes centrales y, por lo tanto, las conversaciones con ellos no fueron grabadas. Este hecho pudo haberles dado a algunos mayor libertad para expresar burla, prejuicios de género o de otro tipo, o simplemente indiferencia ante el problema. Cabe destacar, sin embargo, que algunos de estos "intermediarios" se mostraron conscientes o sensibles al problema. Las conversaciones mantenidas con ellos y registradas en los diarios de campo aportaron elementos muy valiosos para la comprensión de los discursos que circulan en la sociedad en torno a la violencia. Si bien este discurso no fue el más frecuente entre los informantes directos, se considera justo tomarlo como el discurso "dominante" en la medida en que, al encontrarse muy presente entre quienes detentan posiciones de poder, continúa impregnando no sólo el imaginario social, sino también el nivel de la formulación de políticas y de organización de los ámbitos de trabajo. Esto plantea obstáculos para una mejor atención de la violencia en los servicios, en la medida en que bloquea el avance de los discursos más progresistas presentes entre un grupo importante de prestadores de salud.

\section{Discurso antiviolencia basado en valores tradicionales de género}

Este podría llamarse el grupo de "las empáticas". Quienes se adhirieron a él fueron, en su totalidad, mujeres. En este tipo de discurso se reconoce la existencia de la violencia, y se expresa que ésta se puede manifestar de diversas formas (por medio del control económico, de insultos, de la manipulación psicológica o por la imposición de actividades sexuales en contra de la voluntad de la mujer). Se le ve como parte de un sistema en el que participan muchas personas: padres y madres que envían mensajes de dominación masculina a sus hijos; compañeros que presionan a otros para que sometan a sus parejas; profesionales que se rehúsan a atender el problema o que victimizan a las personas que buscan atención. Se piensa, asimismo, que la violencia tiene su origen en ciertas concepciones tales como el "machismo" o "que la mujer se debe quedar en su casa". Se considera que la violencia conyugal es un problema que, de forma directa o indirecta, presente o potencialmente, afecta a todos, incluyendo a las emisoras del discurso, quienes se sienten afectadas por la problemática debido a una identificación de género ("me da mucho coraje como mujer..."). El tono del discurso tiende a ser emocionalmente fuerte, incluyendo desde compasión hasta coraje hacia la víctima, por permitir el maltrato, e impotencia por el deseo de ayudar a romper estos círculos de violencia y por la dificultad percibida para poder hacerlo.

Como se señaló, el grupo que se adscribe a este tipo de discurso está conformado exclusivamente por personal de sexo femenino, predominando en él las enfermeras y las trabajadoras sociales. Frecuentemente se identifican con las mujeres violentadas, ya que se ven a sí mismas como miembros del mismo grupo. Así, la victimización de otra mujer es sentida como la derrota de un miembro del grupo de pertenencia, derrota que se percibe, además, como evitable. Es un discurso que condena la violencia, pero tiende a hacerlo también desde un marco valorativo tradicional que otorga gran valor al papel de la mujer como madre, esposa y encargada de cuidar a los otros y de mantener la armonía familiar. En tanto encarnación de estas virtudes, la mujer no merece ser maltratada sino, por el contrario, recibir de parte del hombre un trato "caballeroso".* Si esto no ocurre, suele considerarse que se trata de un problema de comunicación que ella puede resolver ayudando a su pareja a comunicarse mejor. Llama la atención la invisibilidad del agresor, aun en este grupo que se muestra muy sensible al problema. Dado que la mayoría de quienes se adscriben a este discurso son trabajadoras sociales y enfermeras, se podría suponer que, además de la identificación de género y de la cercanía con las pacientes, la preocupación por las mujeres maltratadas está relacionada también con su perfil profesional, que es coherente con esta concepción de la feminidad: el trabajo social y de enfermería es percibido con frecuencia como uno en cuyo núcleo se encuentra el cuidado y la protección de otros. Se piensa que es tarea de la mujer solucionar los problemas emocionales y familiares, entre ellos el problema de la violencia. De esta manera, el discurso dominante y el discurso antiviolencia comparten algunos

\footnotetext{
* Parte de este discurso tradicional de género es la prescripción moral según la cual a la mujer virtuosa "no se la toca ni con el pétalo de una rosa".
} 
elementos, aun cuando los emisores de uno y otro tengan actitudes diferentes e incluso opuestas hacia quienes sufren violencia.

En cuanto al nivel pragmático, el motivo más mencionado de la inacción frente a la violencia en este grupo es el temor. Se teme a posibles "represalias" que son percibidas de manera difusa, a veces atribuidas al agresor ("en este centro, donde trabajamos todas mujeres, puede venir el esposo de la paciente y golpearnos a nosotras"); otras veces a agentes institucionales ("me pueden regañar si pierdo mi tiempo en esto en lugar de hacer mi trabajo") o, simplemente, ser acusadas de "feministas" por sus colegas. Otro motivo esgrimido es la impotencia por falta de apoyo institucional (tanto de salud como de otras instancias) o, incluso, por falta de apoyo de la propia mujer maltratada que, con frecuencia, se arrepiente de tomar acciones para salir del problema. Algunas también mencionan la "pena ajena" como motivo para no indagar en la situación de la víctima. Suelen definir su situación de impotencia con la metáfora de "estar entre la espada y la pared", es decir, tener la obligación ética de atender el caso, notificarlo y eventualmente denunciarlo, pero al mismo tiempo no contar con el apoyo de las propias víctimas que, "después de tres días, de enojo, se reconcilian con el agresor y no quieren saber más nada".

\section{Discurso antiviolencia basado en algunas ideas sobre "lo moderno"}

Otras nociones que coexisten con el discurso antiviolencia, y con más frecuencia que con las nociones tradicionales recién mencionadas, provienen de ciertas ideas sobre "lo moderno" vinculadas con la educación y la movilidad social a ella asociada. A este grupo podría llamársele el de "los educados". En este discurso se identifica a la violencia con valores y formas de organización familiar y de educación propios del pasado; también con la concepción según la cual la mujer debe complacer al hombre en todo, con roles femeninos hogareños y subordinados, con una organización familiar en la que la mujer quedaba a expensas de la familia del marido y aislada de sus propias redes, sin recursos mentales, porque se le enseñaba que así era la normalidad de las cosas, para emanciparse económica y socialmente de la situación. En estos casos, se afirma que el problema es menos frecuente entre mujeres jóvenes y educadas. Pero en este grupo, estas nociones pueden ser coherentes tanto con el discurso antiviolencia como con prejuicios étnicos o de clase ("estas cosas suceden con más frecuencia en los sectores rurales") o regionales ("el problema se da entre los inmigrantes"). El emisor del discurso se identifica a sí mismo con la modernidad, enemiga de la violencia, e identifica a representantes de la "otredad" social como parte de ese pasado sumergido en la violencia ("no son de este estado", "vienen huyendo", "la gente de zonas rurales es agresiva", tienen "problemas mentales", etcétera). Aquí se incluyen las creencias del informante sobre la "explicación" (y a veces justificación) de la violencia, incluyendo causas psicológicas, morales, sociales, culturales y de género. Suelen aparecer respuestas del tipo "son ignorantes" o "les falta educación". Estas concepciones coinciden con las señaladas en el estudio ya citado sobre personal de salud ${ }^{11}$ acerca de que la violencia es algo que les sucede a otros, generalmente personas con menos educación que ellos mismos.

Este grupo está conformado por la gran mayoría de los médicos de ambos sexos y por algunas enfermeras y trabajadoras sociales. En términos pragmáticos, estos prestadores no se desentienden del problema como lo hacen los del primer grupo, pero tampoco dan seguimiento a los casos. Su conducta más típica en los casos visibles de violencia es canalizar a las mujeres a trabajo social o a psicología donde, a su vez, la usuaria puede recibir un maltrato adicional o bien un trato "empático", pero esto, sin duda, marca una diferencia que puede tener resultados de largo plazo, aun cuando diste de ser un tratamiento integral.

\section{Discurso antiviolencia basado en los derechos de la mujer}

Finalmente, el discurso denominado "antiviolencia basado en los derechos de la mujer", también forma parte de una "ideología de la modernidad" pero en sus aspectos éticos más fundamentales, en la medida en que manifiesta conciencia y compromiso con los derechos y dignidad de las mujeres, independientemente de su papel en la familia y de su posición social. Por esta razón, podrían llamarse los "progresistas" o los "modernos propiamente dichos". En este grupo prevalece la visión de la violencia como delito y la defensa de la víctima como sujeto de derechos. Existe una mayor tendencia al compromiso personal, que puede ir, desde el apoyo moral y los consejos, hasta el acompañamiento de la mujer ante instancias de denuncia. No obstante, y en relación con el nivel pragmático, la discontinuidad de estas acciones suele estar justificada con el argumento de la impotencia, muy similar al ya mencionado para el segundo grupo.

De manera sorprendente, ya que existe el prejuicio de que el personal de salud suele ser "insensible" ante la violencia contra la mujer, se encontró que éste es, con mucha diferencia, el grupo más numeroso, donde predominan los trabajadores sociales pero con una presencia importante también de médicos y enfermeros de ambos sexos y algunas psicólogas. La edad tien- 
de a ser menor en este grupo y son más numerosos aquellos que trabajan en servicios de urgencias.

\section{Discusión}

\section{El perfil de los prestadores y su adhesión a los diferentes discursos}

Sin duda, estos discursos no existen en "estado puro". Son construcciones teóricas a partir de universos valorativos que circulan en la sociedad y que encarnan en los sujetos de manera compleja. Un mismo individuo puede haber incorporado elementos discursivos diversos - de acuerdo con su posición social, género, profesión, edad o experiencia personal-, que suelen "activarse" ante determinados estímulos, situaciones o preguntas. Por ejemplo, es frecuente encontrar en muchas prestadoras de salud la coexistencia de una identificación de género (cercanía con las víctimas) y, al mismo tiempo, el establecimiento de distancias sociales ("no soy como ellas porque tengo estudios y trabajo"; "yo no me dejo"; "en mi casa eso no pasa"), es decir, una combinación de los discursos 2 y 3, vinculada con la ambigüedad que sienten muchas mujeres frente a la violencia y con un mecanismo de distanciamiento frecuente entre quienes se sienten socialmente cerca de un grupo estigmatizado y desean establecer límites con el mismo. En este caso, se trata de la identificación con las víctimas en tanto mujeres -y con el abusador en el caso de los hombres- pero, a la vez, del temor de ser ubicados en el mismo grupo social, señalando la diferencia en educación y estatus como marcador del límite. De manera similar, otro estudio sugirió que la experiencia de abuso que hubiera sufrido una enfermera no afectaba necesariamente su actitud hacia el mismo, pero sí la probabilidad de identificarse con la mujer maltratada. ${ }^{20}$ Otro ejemplo de deslizamiento de este tipo es el de algunas psicólogas, que si bien, como mujeres, pueden expresar empatía hacia las víctimas (discursos 2 y 4), ante determinadas preguntas comienzan a hablar como profesionistas y a dar explicaciones psicologizantes, centrando su atención en la responsabilidad de la mujer por permanecer en el vínculo violento, ya sea por "obtener beneficios psicológicos" de la violencia, por "instinto de muerte", masoquismo o cualquier otro tipo de desorden mental, muchas veces tácitamente asociado a determinados grupos sociales (elementos de los discursos 1 o 3 ).*

\footnotetext{
* Esto puede indicar que los enfoques que incluyen aspectos sociales y de poder en las relaciones entre géneros no han permeado aún el discurso psicológico que se enseña en las universidades, a pesar de que en las publicaciones actuales de todas las corrientes están cada vez más presentes.
}

Al prestar atención entonces a estas posibles combinaciones de elementos discursivos, y con fines analíticos, se clasificó a los informantes en alguna de las cuatro categorías, teniendo en cuenta el discurso al que más se acercaba cada uno. Así, se observó que la adhesión más pronunciada a uno $\mathrm{u}$ otro discurso estaba relacionada con el género (en el grupo 2 todas son mujeres); la profesión (la mayoría de los médicos de ambos sexos está en el grupo 3); la edad (en el grupo 4 el promedio de edad desciende); el tipo de servicios donde trabajan (quienes lo hacen en urgencias y en trabajo social, donde los casos de violencia son más visibles, tienden a adherir al discurso 4) y el compromiso de la institución donde trabajan (es más común el discurso 4 entre quienes se sienten apoyados por colegas o directivos de la institución donde se desempeñan).

En cuanto al género, muchas mujeres entrevistadas expresaron que el personal masculino se negaba a reconocer la existencia del problema. Mientras que un grupo importante de ellas tendió a ver la violencia conyugal como algo que les afectaba real o potencialmente de forma directa, los hombres que aceptaron la entrevista tendieron a hablar del tema -independientemente de su mayor o menor sensibilidad al mismocomo si fuera algo ajeno a ellos. Es importante mencionar que el personal médico masculino estuvo menos dispuesto a ser entrevistado sobre el tema que el femenino, y que, incluso, algunos de ellos manifestaron nerviosismo y rechazo ante la invitación a hablar de la violencia de pareja.

Con respecto a la profesión, es interesante notar una dicotomía conceptual todavía muy presente en los prestadores de salud, entre aquellos que consideran los "males del cuerpo" (que corresponde tratar a los médicos y tal vez enfermeros/as) en contraposición con los "males del alma", de los que deben ocuparse los psicólogo/as y eventualmente las trabajadoras sociales. Muchos de los que no se involucran en el problema aducen que eso "no les compete" porque "no son psicólogos", mientras que entre quienes sí lo hacen, varios lo justifican con frases como "es que yo tengo alma de psicólogo/a" o "yo le hago medio de psicólo$\mathrm{ga} / \mathrm{o}$ con las pacientes". Incluso los psiquiatras se diferencian a sí mismos de los psicólogos (y viceversa), ubicándose más cerca de los médicos en términos de estatus profesional y discurso sobre la violencia. Se observa que no es sólo la cercanía al problema (de quienes tienen que "ver" los casos) lo que hace más sensibles a los prestadores, sino también su definición del tipo de problemas que atiende cada uno y, por lo tanto, su idea del rol profesional. De este modo, tanto en el grupo donde prevalece el discurso llamado "dominante, basado en estereotipos rígidos de género" (1), como en el que adhiere al denominado "antiviolencia, 
basado en algunas ideas sobre lo moderno" (3), la indiferencia o inacción frente a la violencia contra las mujeres es justificada con el argumento de que el problema no es de la competencia profesional del informante. Las respuestas típicas son: "eso no nos corresponde a nosotros"; "es tarea de un médico legista"; o bien, "yo no soy psicólogo/a para atender esos casos".

\section{EI nivel institucional}

Es en este punto que el nivel institucional adquiere una especial relevancia. Como se ha mencionado, los trabajadores de la salud pueden haber internalizado con mayor o menor fuerza distintos tipos de discurso, pero una serie de factores contextuales pueden fortalecer o debilitar la distribución de los mismos. Hacia el interior de las instituciones, las relaciones laborales que favorecen una estratificación rígida y una falta de comunicación entre el personal de la institución, así como las políticas de trabajo que favorecen una "visión de túnel" $21, *$ de las profesiones (con las diferencias de prestigio que esto suele conllevar), dificultan el acercamiento laboral a la violencia. En las pocas instituciones donde hubo mayor comunicación, una estratificación menos rígida al interior de la institución y una menor visión de túnel profesional, también se observó que a las personas les resultaba más fácil sostener los elementos discursivos relacionados con la defensa de los derechos de la mujer. Lo anterior se hizo evidente en las pocas unidades de salud donde existían grupos de autoayuda para mujeres víctimas de violencia, en los que se registraba un buen trabajo interinstitucional, un interés de parte de la directiva y una participación de personas que no formaban parte de la clínica. ${ }^{\ddagger}$

En cuanto a los elementos externos a las instituciones del sector, los prestadores notificaron una

\footnotetext{
* La expresión "visión de túnel" es utilizada por algunos psicoterapeutas para referirse a cierta unidireccionalidad en la percepción de los problemas del paciente, debido a que la interacción con éste es guiada por una serie limitada de ideas (referencia 21). La baja percepción de los efectos de la violencia en las pacientes por parte de algunos médicos, podría estar relacionada, en parte, con una visión de túnel originada en su preparación y expectativas profesionales dirigidas únicamente al ámbito biológico, más allá del cual sienten que no es competencia de su profesión. Algo semejante se observó entre muchos psicólogos, cuya visión estaba dirigida únicamente al ámbito intrapsíquico y, en algunos casos, dentro de éste, hacia las dimensiones psicopatológicas. A estos profesionales les cuesta trabajo reconocer la existencia de dimensiones sociales, como el poder, la estratificación, los roles sociales y demás.

‡ Por ejemplo un grupo de psicólogas que trabajan de forma voluntaria y dan seguimiento a casos de violencia, o algunas ONG que ofrecen información o capacitan al personal, donde las había.
}

desconfianza generalizada en todo lo referente a cuestiones legales, y en la falta de mecanismos integrales en la sociedad en general para lidiar con el problema de la violencia doméstica. ${ }^{*}$ Un tratamiento adecuado e integral de la violencia en los servicios de salud, según muchos prestadores, debería fomentar el seguimiento posterior y la canalización a lugares adecuados donde las mujeres reciban apoyo legal psicológico para la recuperación de su autonomía económica.

Aparentemente, el desconcierto para tomar acciones en contra de la violencia derivado de los factores recién mencionados, fortalece a su vez los tres primeros tipos de discurso, los cuales contienen elementos que pueden ser coherentes con la justificación de la inacción frente a esta.

De esta manera, se observa que no es el género o la profesión del prestador de servicios de manera aislada lo que determina su conducta frente a la violencia (aunque estos factores están, sin duda, relacionados: en un contexto global de escaso compromiso institucional frente al problema, que tiende a homogeneizar las prácticas profesionales y a desmotivar la acción), sino la mayor o menor adhesión a universos valorativos diferentes -aunque muchas veces mezclados y articulados de manera compleja con esos y otros factores-, lo que determina las diferencias que pudieran encontrarse entre sus prácticas cotidianas y, especialmente, su manera de vivir y enfrentar la violencia contra la mujer.

\section{Conclusiones}

Se puede suponer que la adscripción a uno u otro tipo de discurso sobre la violencia puede tener importantes implicaciones para su atención, tanto entre los profesionales como entre los individuos, así como en lo tocante a las relaciones entre los distintos sectores dentro de las instituciones, a las relaciones entre éstas y diversos sectores de la población, y en cuanto a las políticas institucionales.

Estas conclusiones tienen importancia clave en el diseño de intervenciones dirigidas a revertir las actitudes de los prestadores, en la medida en que permiten identificar aquellos puntos o elementos discursivos específicos por grupo o comunes a todos, donde sea factible dirigir mensajes que transformen la manera en que cada uno tiene de comprender y acercarse al problema de la violencia y de quienes la padecen.

\footnotetext{
* Verbigracia protección policíaca para las víctimas, existencia de refugios, directrices legales claras para los trabajadores de salud, métodos de rehabilitación laboral y psicológica para las mujeres que tratan de salir de la violencia, entre otros elementos.
} 
Asimismo, los esfuerzos deben estar dirigidos a transformar elementos que, dentro y fuera de las instituciones de salud, evitan que el problema de la violencia de pareja pueda ser afrontado de forma adecuada. Dado que el discurso más reacio a reconocer a la violencia conyugal como problema -y por lo tanto a darle respuesta- está muy presente entre autoridades y personal de puestos jerárquicos, las estrategias de intervención para involucrar más al sector salud y a otros en este problema deben tener en cuenta a este grupo en particular, que al detentar posiciones de poder desalienta a los prestadores más dispuestos a atender los casos de violencia de manera eficaz. Es decir, para atacar al mismo tiempo tanto la negación de unos como la impotencia de otros frente a la violencia, es clave no sólo intervenir en el imaginario de los cuadros directivos, sino también fomentar el diálogo entre profesionales de distinta formación al interior de las unidades de atención, y crear canales de cooperación intersectorial que alienten y permitan dar cauce a la buena disposición de muchos prestadores, sin dejar de tener una política integral de capacitación y difusión de la normatividad oficial dirigida a todo el personal de salud.

\section{Referencias}

I. Heise L, Pitanguy J, Germain A.Violencia contra la mujer. La carga oculta sobre la salud. Washington DC: OPS, 1994.

2. Family violence prevention project. An informational packet for health professionals. San Francisco, California: Family violence prevention project 1993.

3.Viterbo-Silva I.Violência contra mulheres: a experiência de usuárias de um serviço de urgência e emergência de Salvador, Bahia Brasil. Cad Saude Publica 2003;19 suppl 2:S263-S272.

4. Sugg N, Inui T. Primary care physicians' response to domestic violence. Opening Pandora's Box. J Am Med Assoc 1992;267 (23): 3157-3160. 5. Sampselle CM. The role of nursing in preventing violence against women. J Obstet, Gynecol Neonatal Nursing 199|;20(6): 48|-487.
6. Agoff C, Rajsbaum A, Herrera C. Perspectivas de las mujeres maltratadas sobre la violencia de pareja en México. Salud Publica Mex 2006;48 supl 2:S307-S3|4.

7. Langford DR. Policy issues for improving institutional response to domestic violence.J Nurs Adm 1996; 26 (I): 39-45.

8. Campbell JC. Battered women's experiences in the emergency department. J Emerg Nurs 1994; 20(4): 280-288.

9. Hoff LA, Ross M.Violence content in nursing curricula: Strategic issues and implementation. J Adv Nurs 1995; 21:137-142.

10. De Barbieri T. Sobre la categoría género: una introducción teóricometodológica. Isis Internacional. Ediciones de las Mujeres 1992; I7: I II128.

I I. Kim J, Motsei M. "Women enjoy punishment": attitudes and experiences of gender-based violence among PHC nurses in rural South Africa. Soc Sci Med 2002;54: 1243- 1254.

12. SSA / INSP. Encuesta Nacional sobre Violencia contra las Mujeres 2003 (ENVIM 2003). Cuernavaca, Morelos: Instituto Nacional de Salud Pública, 2003.

13. Glaser B, Strauss A. The discovery of grounded theory. Strategies for qualitative research. Chicago: Aldine, 1967.

14. Strauss A, Corbin J. Basics of qualitative research. Newbury Park: Aldine, 1990.

I5. Méndez-Hernández P,Valdez-Santiago R,Viniegra-Velázquez L, Rivera-Rivera L, Salmerón Castro J.Violencia contra la mujer: conocimiento y actitud del personal médico del Instituto Mexicano del Seguro Social, Morelos, México. Salud Publica Mex 2003; (5) 6: 472-482. 16. SSA / INSP Informe ejecutivo de la Encuesta Mujer y Violencia en México. México: INSP/SSA, 2004.

17.Valdez-Santiago R. Respuesta médica ante la violencia que sufren las mujeres embarazadas. En: Marta Torres, comp. La violencia contra las mujeres en contextos urbanos y rurales. México: El Colegio de México, 2004.

18. Fawcett G. Manejo de víctimas de violencia doméstica: desarrollo y evaluación de un programa dirigido al personal de salud. Docs. de Trabajo N 26, Population Council / INOPAL III: México, 1998. 19. SSA. Norma Oficial Mexicana NOM-190-SSAI-1999. Prestación de servicios de salud. Criterios para la atención de la violencia familiar. México: Secretaría de Salud, 1999.

20. Moore ML, Zaccaro D, Parsons LH. Atitudes and practices of registered nurses toward women who have experienced abuse/ domestic violence. J Obstet Gynecol Neonatal Nursing 1998; 27 (2): 175-182.

21. Bellak L, Siegel H. Manual de psicoterapia breve, intensiva y de urgencia. México: El Manual Moderno, 1986. 